\title{
Auf dem Weg zur Union in der Union. Institutionelle Auswirkungen der differenzierten Integration in der Eurozone auf die EU
}

\author{
Nicolai von Ondarza*
}

Ursprünglich als letztes Mittel der europäischen Integration eingeführt, hat die Nutzung differenzierter Formen der Zusammenarbeit, an denen sich nur Teile der EU-Mitgliedstaaten beteiligen, in der Europäischen Union zuletzt deutlich zugenommen. Auf primärrechtlicher Ebene wurden mit dem Vertrag von Lissabon mehrere neue Opt-Outs für einzelne Mitgliedstaaten wie die Ausnahme von der Anwendbarkeit der Charta der Grundrechte der Europäischen Union für Großbritannien und Polen eingeführt. Auch in der Sicherheits- und Verteidigungspolitik sowie der Innen- und Justizpolitik wurden neue Sonderformen der flexiblen Zusammenarbeit in den Vertrag aufgenommen. In der politischen Praxis haben die Mitgliedstaaten 2010/2011 für die Regelungen zu transnationalen Scheidungen (15 Teilnehmer) und dem gemeinsamen EU-Patent (25) zudem erstmals auf das Instrument der Verstärkten Zusammenarbeit zurückgegriffen. Vor allem aber gestalteten die Staats- und Regierungschefs im Rahmen der Bewältigung der Schuldenkrise mit dem Euro-Plus-Pakt (23) und dem außerhalb des Primärrechts stehenden Fiskalpakt (25) in kurzer Abfolge um die Eurozone neue Formate differenzierter Integration mit unterschiedlicher Zusammensetzung. Die Bankenaufsicht und die Finanztransaktionssteuer sind weitere Projekte, an denen sich nur Teile der EU-Mitgliedstaaten beteiligen wollen und die bereits auf den Weg gebracht wurden. Das Europa der mehreren Geschwindigkeiten ist damit längst Realität geworden.

Diese differenzierte Integration verändert die politischen Dynamiken und Prozesse in der Europäischen Union. In aller Öffentlichkeit zeigen sich diese Veränderungen und die damit verbundenen Befürchtungen über eine ,Spaltung Europas ‘ in den Diskussionen während der Schuldenkrise in der Eurozone. Die zentralen Entscheidungen nicht nur über die finanzielle Unterstützung der Krisenstaaten, sondern auch über Reformen in der wirtschaftspolitischen Steuerung der gesamten Europäischen Union wurden größtenteils im Rahmen der Eurostaaten getroffen. ${ }^{1}$ Mit den nunmehr institutionalisierten Eurogipfeln und einem eigenen ständigen Präsidenten wird die Sonderstellung der Eurostaaten stetig ausgebaut. Nicht zuletzt zur Entschärfung einer „Teilung der EU in die Länder der Eurozone und die Länder draußen" 2 beteiligten sich selbst Nicht-Eurostaaten freiwillig an Teilen der Finanzhilfen sowie dem Euro-Plus-Pakt und dem Fiskalpakt. Als ein möglicher Ausweg aus der Krise werden zunehmend auch weitergehende Integrationsprojekte der ,Euro-17` plus weitere interessierte Staaten diskutiert.

Dennoch stehen sich in der politischen Diskussion über die Nutzung differenzierter Integration weiterhin zwei Gegenpole unversöhnlich gegenüber: Auf der einen Seite preisen Befürworter die Differenzierung als Strategie, mit der die Integration der Europäischen Uni-

* Dr. Nicolai von Ondarza, Stiftung Wissenschaft und Politik, Berlin.

1 Hierzu gehörten etwa die Entscheidungen zum Aufbau des Eurorettungsschirms (März 2010), zur Begründung des Euro-Plus-Pakts (März 2011), zum zweiten Griechenlandrettungspaket (Juli 2011) und zum Fiskalpakt (März 2012).

2 So die Formulierung des polnischen Ministerpräsidenten Tusk. Vgl. Frankfurter Allgemeine Zeitung: Vor dem EU Gipfel: ,Deutschland diktiert niemandem etwas', 16.12.2010. 
on vorangetrieben werden kann, in dem sich die besonders integrationswilligen Staaten zusammenschließen. ${ }^{3}$ Auf der anderen Seite sehen Kritiker in der differenzierten Integration eine fundamentale Bedrohung für den Zusammenhalt der Europäischen Union, in dem nichtbeteiligte Mitgliedstaaten ausgegrenzt werden und langfristig die Spaltung der Union in Kauf genommen wird. ${ }^{4}$ Angesichts dieser auf beiden Seiten stark normativ aufgeladenen Diskussion ist es umso dringender, die tatsächlichen Folgen der differenzierten Integration auf die EU-Institutionen und das institutionelle Gleichgewicht in der Union empirisch zu analysieren.

\section{Die Schuldenkrise treibt die differenzierte Integration voran}

Die Europäische Union hat sich mittlerweile weit auf den Pfad der differenzierten Integration begeben. Die Mitgliedstaaten haben dafür eine breite Palette von Instrumenten geschaffen, die sich jedoch in ihren rechtlichen Möglichkeiten und Schranken deutlich unterscheiden. Das formelle Instrument der Verstärkten Zusammenarbeit erlaubt zwar die vollständige Nutzung des institutionellen Rahmens der Europäischen Union und lässt sich seit dem Vertrag von Lissabon in allen Politikbereichen anwenden. Gleichzeitig sind der Verstärkten Zusammenarbeit aber enge Schranken auferlegt, sodass sie weder zur Kompetenzerweiterung noch zum Austritt aus bereits integrierten Bereichen genutzt werden kann, wie es etwa Großbritannien anstrebt. Die nur semantisch verwandte Ständige Strukturierte Zusammenarbeit (SSZ) erlaubt hingegen die Kooperation beim Aufbau militärischer Fähigkeiten, ist bislang aber noch nicht zur Anwendung gekommen. Hinzu kommen für einzelne Mitgliedstaaten im Sinne von negativer Differenzierung im Primärrecht abgesicherte OptOuts aus einzelnen Politikbereichen. Darüber hinaus können EU-Mitgliedstaaten jederzeit außerhalb des Unionsrahmens völkerrechtliche Verträge schließen, solange diese die an die Europäische Union abgetretenen Kompetenzen und die Solidaritätspflicht in der Union nicht berühren. ${ }^{5}$

Auf Basis dieser Grundlagen hat sich ein breitgefächertes System entwickelt, bei dem die Mitgliedstaaten mit Ausnahme der SSZ bereits von allen möglichen Rechtsformen Gebrauch gemacht haben. Die differenzierte Integration war dabei bis dato auf drei Politikbereiche begrenzt: Die Währungsunion mit den permanenten Opt-Outs für Großbritannien und Dänemark, die Innen- und Justizpolitik ebenfalls mit Ausnahmeregeln aus dem Schengener Abkommen und/oder dem Raum der Freiheit, der Sicherheit und des Rechts (RSFR) für Dänemark, Großbritannien und Irland sowie als dritten Politikbereich die Gemeinsame Si-

3 Siehe stellvertretend Sebastian Kurpas/Julia De Clerck-Sachsse/José Torreblanca/Gaëtane Ricard-Nihoul: From Threat to Opportunity: Making flexible integration work, European Policy Institutes Network: EPIN Working Paper 15/2006; Kenneth Dyson/Angelos Sepos: Differentiation as Design Principle and as Tool in the Political Management of European Integration, in: Kenneth Dyson/Angelos Sepos (Hrsg.): Which Europe? The Politics of Differentiated Integration, Basingstoke 2010, S. 3-23; Jean-Claude Piris: The Future of Europe. Towards a Two-Speed Europe. Cambridge 2012.

4 So warnte EU-Kommissionspräsident José Manuel Barroso noch im November 2011 vor jeglicher Form differenzierter Integration: „Let me be clear - a split union will not work. This is true for a union with different parts engaged in contradictory objectives; a union with an integrated core but a disengaged periphery; a union dominated by an unhealthy balance of power or indeed any kind of directorium. All these are unsustainable and will not work in the long term“. José Manuel Durão Barroso: The State of Europe - Die Europa Rede, Berlin, 9 November 2011, SPEECH/11/738.

5 Daniel Thym: Supranationale Ungleichzeitigkeit im Recht der europäischen Integration: in: EuropaRecht 5/2006, S. 637-655 
cherheits- und Verteidigungspolitik (GSVP). ${ }^{6}$ In letzterer hat zwar nur Dänemark ein volles Opt-Out, an vielen einzelnen GSVP-Operationen und Maßnahmen nehmen aber jeweils nur Gruppen von Mitgliedstaaten teil.

Differenzierung ist also kein neues Phänomen der europäischen Integration und ein Europa der mehreren Geschwindigkeiten ist längt Realität der Zusammenarbeit in der Europäischen Union. Die bisherige differenzierte Integration war allerdings eine Sonderform, die nur als letztes Mittel zur Anwendung kam. So war zum einen Konsens, dass trotz der bestehenden Ausnahmeregelungen der politische Rahmen für die europäische Integration von allen EU-Mitgliedstaaten gestaltet wird. Aus diesem Grunde gab es - bis 2010 - beispielsweise keine Sondertreffen der Staats- und Regierungschefs der Eurozone. Bei der großen Erweiterung 2004/2007 mussten sich die neuen Mitgliedstaaten zudem verpflichten, langfristig dem Schengenraum und der Eurozone beizutreten. Zum anderen war die Hemmschwelle groß, tatsächlich zur differenzierten Integration zu greifen. So wurde das formell seit dem Amsterdamer Vertrag existierende Mittel der Verstärkten Zusammenarbeit ebenfalls bis 2010 nicht genutzt. Anwendung fand es höchstens als - abschreckendes - Druckmittel. Beispielsweise gab die italienische Regierung 2001/2002 ihren Widerstand gegen den Europäischen Haftbefehl auf, sobald die Kommission und eine glaubhafte Gruppe von Mitgliedstaaten damit gedroht hatten, den Haftbefehl über die Verstärkte Zusammenarbeit einzuführen. Wenn die differenzierte Integration wie bei dem Schengener Abkommen oder der Einführung des Euros zur Anwendung kam, dann jeweils nur als letztes Mittel um Blockaden in festgefahrenen Verhandlungen zu überwinden.

Diese Hemmschwelle vor dem Einsatz differenzierter Integration ist ab Ende der 2000er Jahre sukzessive gefallen. Schon der Lissabonner Vertrag hat die Anzahl an Opt-Outs für einzelne Staaten massiv ausgeweitet. ${ }^{7}$ 2010/2011 griffen die EU-Mitgliedstaaten dann erstmals zur Verstärkten Zusammenarbeit beim transeuropäischen Scheidungsrecht (zunächst 14, mittlerweile 15 Staaten $)^{8}$ und dem EU-Patentrecht (25 Staaten), weil jeweils lange blockierte Verhandlungen nur in kleineren Gruppen zu Ende geführt werden konnten.

Vor allem verstärkt die seit Anfang 2010 andauernde Schuldenkrise die Rolle der Eurozone innerhalb der Wirtschaftspolitik der Europäischen Union. Neben den reaktiven Maßnahmen auf die Krise in Form von Finanzhilfen für die Krisenstaaten und dem Aufbau der Europäischen Finanzstabilitätsfazilität (EFSF) beziehungsweise dem Europäischen Stabilitätsmechanismus (ESM) wurden im Zuge des Krisenmanagements bedeutende strukturelle Reformen an der wirtschaftspolitischen Steuerung in der Europäischen Union vorgenommen. Hierzu gehören insbesondere das Europäische Semester, das heißt die Koordinierung in der Aufstellung der nationalen Haushalte, sowie das Six-Pack zur Stärkung des Stabilitätsund Wachstumspakts und zur Reduzierung makroökonomischer Ungleichgewichte. Obgleich der Großteil dieser Reformen vor allem die Eurozone betrifft, sind sie nicht ohne Wirkung auf die Nicht-Euro-Mitglieder. Dennoch wurden die zentralen Entscheidungen jeweils in der Eurogruppe beziehungsweise Sondertreffen der Staats- und Regierungschefs der

6 Für einen aktuellen Überblick über die Projekte differenzierter Integration im EU-Rahmen siehe Nicolai von Ondarza: Zwischen Integrationskern und Zerfaserung. Folgen und Chancen einer Strategie differenzierter Integration, Stiftung Wissenschaft und Politik: SWP-Studie S 20/2012, S. 11, 35.

7 Janis A. Emmanouilidis: Conceptualizing a Differentiated Europe, Hellenic Foundation for European \& Foreign Policy: Eliamep Policy Paper 10/2008, S. 31.

8 Litauen hat sich 2012 nachträglich der Verstärkten Zusammenarbeit im Bereich des Scheidungsrechts angeschlossen. 
Eurozone vorbereitet und politisch ausgehandelt. ${ }^{9}$ Nicht-Euro-Mitgliedstaaten blieben bei diesen Treffen entweder außen vor oder waren nur am Rande beteiligt, obwohl zentrale Weichen für die wirtschaftliche Entwicklung der Europäischen Union als Ganzes gestellt wurden. Zusätzlich haben rechtlich außerhalb des EU-Rahmens 23 EU-Mitgliedstaaten den Euro-Plus-Pakt geschaffen, um sich in der Wirtschaftspolitik enger zu koordinieren, und 25 EU-Mitgliedstaaten mit dem Fiskalpakt eine stärkere Haushaltskontrolle vereinbart. ${ }^{10}$

Die Europäische Union spaltet sich so zunehmend in drei voneinander getrennte Gruppen: Dies sind zum einen die ,Euro-17‘, die sich an allen Rettungs- und Reformmaßnahmen im Rahmen der Krise beteiligen müssen beziehungsweise selbst finanziell unterstützt werden. Die zweite Gruppe umfasst diejenigen Staaten, welche sich wie Polen vertraglich verpflichtet haben, den Euro einzuführen, und dieses Ziel auch weiterhin politisch verfolgen. ${ }^{11}$ Doch bereits innerhalb dieser Gruppe der ,Pre-Ins' variiert die Bereitschaft, sich am Eurokrisenmanagement und zukünftiger Vertiefung zu beteiligen, erheblich. Hinzukommt Dänemark, welches zwar ein dauerhaftes Opt-Out aus dem Euro hat, sich aber freiwillig an Reformmaßnahmen (Euro-Plus-Pakt, Fiskalpakt) in der wirtschaftspolitischen Steuerung beteiligt. Eine dritte Gruppe bilden die dauerhaften Außenseiter, die entweder ein Opt-Out haben (Großbritannien) oder sich wie Schweden und zunehmend auch Tschechien der rechtlichen Verpflichtung durch Nicht-Erfüllung der Beitrittskriterien entziehen. Angesichts dieser Konstellation ist die Spaltung dauerhafter Natur und wird sich mit jedem Schritt zu mehr Integration in der Eurozone weiter verschärfen.

Schon in naher Zukunft dürfte die Differenzierung um die Eurozone also weiter zunehmen: Im Oktober 2012 legte die Kommission auf Vorschlag von elf Staaten, die dem Euro angehören, eine Initiative für eine Verstärkte Zusammenarbeit zur Einführung einer Finanztransaktionssteuer vor. ${ }^{12}$ Die im Rahmen des Schuldenkrisenmanagement entwickelte Bankenaufsicht wird zunächst auf die Staaten der Eurozone aufbauen, aber weiteren interessierten EU-Mitgliedstaaten offenstehen. ${ }^{13}$ Während Großbritannien eine Teilnahme klar ablehnt, zeigen sich vor allem viele Pre-Ins einer freiwilligen Einbindung in die Bankenaufsicht gegenüber offen. Nicht zuletzt verhandeln die Mitgliedstaaten der Eurozone bereits über weitere, weitgehende Schritte hin zu einer ,echten Wirtschafts- und Währungsunion', für die verschiedene neue Formen der engeren Zusammenarbeit der Eurostaaten im Gespräch sind. ${ }^{14}$ Auf der anderen Seite hat der britische Premierminister David Cameron im Januar 2013 angekündigt, zusätzliche Ausnahmeregelungen für Großbritannien durchsetzen zu wollen. ${ }^{15}$ Die Europäische Union bewegt sich damit aktuell auf die Schwelle zu, in der differenzierte Integration nicht mehr als die Ausnahme vom Einheitsprinzip zu betrachten ist,

9 Nicolai von Ondarza: Koordinatoren an der Spitze. Politische Führung in den reformierten Strukturen der Europäischen Union, Berlin 2011.

10 Zur Wirkungsweise und Differenzierung von Fiskalpakt, Euro-Plus-Pakt und Six-Pack, siehe Friedrich Heinemann/Marc-Daniel Moessinger/Steffen Osterloh: Feigenblatt oder fiskalische Zeitenwende? Zur potenziellen Wirksamkeit des Fiskalvertrags, in: integration 3/2012, S. 167-182.

11 Konstanty Gebert: A place at the top table? Poland and the Euro crisis, European Council on Foreign Relations: ECFR Reinventing Europe Paper, 2012.

12 Europäische Kommission: Vorschlag für einen Beschluss des Rates über die Ermächtigung zu einer Verstärkten Zusammenarbeit im Bereich der Finanztransaktionssteuer, KOM (2012) 631.

13 Europäische Kommission: Mitteilung der Kommission an das Europäische Parlament und den Rat. Fahrplan für eine Bankenunion, KOM (2012) 510.

14 Europäischer Rat-Der Präsident: Auf dem Weg zu einer echten Wirtschafts- und Währungsunion. 6. Dezember 2012.

15 David Cameron: EU speech at Bloomberg, 23.1.2013. 
sondern zumindest rund um die Eurozone zum bestimmenden Modus Operandi der Integration wird.

\section{Die institutionellen Folgen der differenzierten Integration}

Diese Ausweitung differenzierter Integration wirkt sich auch massiv auf das politische System der Europäischen Union aus. Angesichts der unterschiedlichen Mitgliedschaften der EU-Mitgliedstaaten in zunehmend mehr politischen Projekten sind alle EU-Organe mit der Herausforderung konfrontiert, ob und wie sie ihre Beratungs- und Entscheidungsverfahren an die differenzierte Union anpassen. Dabei ergeben sich erhebliche Folgen für das institutionelle Gleichgewicht der Europäischen Union sowie die Machtbeziehungen zwischen den Mitgliedstaaten. Vor allem die neu entstehenden Doppelstrukturen um die Eurozone stärken in ihrer aktuellen Ausprägung sowohl das intergouvernementale Element in der Entscheidungsfindung als auch den Einfluss besonders der großen Eurostaaten.

\section{Der modulare Rat}

Die institutionelle Anpassung des Rates der Europäischen Union und des Europäischen Rates an Projekte differenzierter Integration ist auf den ersten Blick am einfachsten umzusetzen: Als Organe, in dem die Regierungen ihre Mitgliedstaaten direkt vertreten sind, können hier in jedem einzelnen Entscheidungsverfahren die Beteiligungs- und Mitbestimmungsrechte der nationalen Vertreter rechtlich und politisch klar angepasst werden. So haben beispielsweise bei Entscheidungen über den RFSR nur diejenigen Mitgliedstaaten im Rat der Europäischen Union Stimmrecht, die auch an dem Politikbereich beteiligt sind. ${ }^{16}$ Für Ratsabstimmungen ist es rechtlich unerheblich, ob der Rat sich für einen Politikbereich aus 17 (Eurozone), 24 (RFSR) oder 27 (Europäische Union) Staaten zusammensetzt.

Politisch umstritten an dieser modularen Zusammensetzung des Rates ist nur die Frage, inwieweit Vertreter nicht-beteiligter Mitgliedstaaten in die Beratungen eingebunden werden sollten. Eine solche Teilnahme macht auf der einen Seite die differenzierte Integration transparent, da die Außenseiter jederzeit über den Stand der Beratungen informiert sind und sich sogar informell mit eigenen Initiativen einbringen können. Dies vereinfacht auch einen späteren Beitritt in ein Differenzierungsprojekt, wie es etwa die Verstärkte Zusammenarbeit vorsieht. ${ }^{17}$ Auf der anderen Seite kann eine solche Beteiligung die Beratungen erschweren, insbesondere wenn es um sensible politische Entscheidungen wie in der Eurozone geht, die potenziell alle EU-Mitgliedstaaten zumindest indirekt betreffen können.

Ein Überblick über die bestehenden Formen differenzierter Integration in der Europäischen Union zeigt hier eine klare Trennlinie. Mit der bedeutenden Ausnahme der Eurostrukturen (siehe unten) funktioniert der Rat der Europäischen Union in Projekten differenzierter Integration nach dem Inklusionsprinzip: Nicht-beteiligte Mitgliedstaaten verfügen zwar über kein Stimmrecht, dürfen aber von den Ratsarbeitsgruppen bis hin zum Europäischen Rat auf allen Ebenen des Ratssystems an den Beratungen als Beobachter teilnehmen. Diese Regelung gilt sowohl für die institutionalisierten Formen differenzierter Integration wie die Verstärkte

16 So werden insbesondere im RFSR je nach Nutzung der Opt-In-Rechte von Dänemark, Großbritannien und/oder Irland die Abstimmungsrechte im Rat angepasst.

17 Eine vollständige Beteiligung aller EU-Mitgliedstaaten an einem früheren Projekt differenzierter Integration ist bisher nur ein einziges Mal bei der Sozialcharta gelungen. 
Zusammenarbeit und die SSZ als auch für Sonderformen wie das Schengener Abkommen oder die Opt-Outs im RFSR und der GSVP. ${ }^{18}$

Diese hohe Inklusivität reicht bis zur Ausübung der Ratspräsidentschaft - wenn ein Mitgliedstaat den Ratsvorsitz innehat, so trifft dies formell auch auf diejenigen Ratsformationen und Arbeitsgruppen zu, in denen Verhandlungen zu Projekten differenzierter Integration stattfinden, an denen der betreffende Staat nicht beteiligt ist. So hat Dänemark während seiner Präsidentschaft im 1. Halbjahr 2012 auch den Rat für Justiz und Inneres geleitet, obwohl es für Teile der dort getroffenen Entscheidungen ein Opt-Out hat. Mit dieser Modularität ist der Rat der Europäischen Union also vollständig darauf ausgerichtet, die integrierte, gemeinsame Struktur der Europäischen Union aufrecht zu erhalten.

\section{Das separate Euro-Ratssystem}

Einzige große, dafür aber umso bedeutsamere Ausnahme von dieser Regel ist die gemeinsame Währung: Schon seit Ende der 1990er Jahre haben die Eurostaaten mit der Eurogruppe ein eigenständiges, zunächst informelles Gremium geschaffen, in dem sich ausschließlich ihre Wirtschafts- und Finanzminister treffen. Hinzukommen der Kommissar für Wirtschaft und Währung sowie nicht zuletzt auch der Präsident der Europäischen Zentralbank (EZB). Im Gegensatz zu den anderen Formaten differenzierter Integration basiert die Eurogruppe aber auf dem Prinzip der Ausschließlichkeit, denn die Nicht-Eurostaaten sind aus ihren Beratungen ausgeschlossen. Dies gilt auch für die Pre-Ins, die sich perspektivisch zu einer Einführung des Euros verpflichtet haben und dementsprechend in der Zukunft auch von allen Beschlüssen der Eurozone betroffen sind. Polen etwa hat daher darauf gedrängt, zumindest in seiner Funktion als Ratspräsidentschaft im zweiten Halbjahr 2011 an den Sitzungen der Eurogruppe teilzunehmen - diese Bestrebungen wurden aber insbesondere auf Betreiben Frankreichs mit Verweis auf die geschlossene Natur der Eurogruppe abgelehnt. ${ }^{19}$

Dabei spielt die Eurogruppe vor allem eine politische Rolle als Vorentscheidungsgremium. Denn obwohl sie mit dem Vertrag von Lissabon institutionalisiert wurde, kann sie rechtlich auch weiterhin keine verbindlichen Entscheidungen treffen. ${ }^{20}$ Diese werden formell durch den Rat für Wirtschaft und Finanzen (ECOFIN) verabschiedet. In der Praxis werden die politischen Vorentscheidungen der Eurogruppe aber vom ECOFIN, der in der Regel nach der Eurogruppe tagt, nur rechtlich ohne Debatte nachvollzogen. Die eigentliche Abstimmung im ECOFIN wird so zur reinen Formalität degradiert: Beispielsweise wurde im Februar 2012 in der Eurogruppe in einer bis tief in die Nacht andauernden Sitzung über das zweite Griechenland-Rettungspaket verhandelt. Erst nach Einigung im Euro-Format fand am folgenden Tag eine Sitzung des ECOFIN statt, in der die politische Vereinbarung ohne Debatte akzeptiert wurde. ${ }^{21}$ Von einem informellen Gremium ist die Eurogruppe damit zu einem der politisch wichtigsten Entscheidungszentren in der Union geworden. ${ }^{22}$

18 Für eine Darstellung der verschiedenen Formen differenzierter Integration, siehe Alexander Stubb: A Categorization of Differentiated Integration, in: Journal of Common Market Studies 2/1996, S. 283-295; Katharina Holzinger/Frank Schimmelfennig: Differentiated Integration in the European Union: Many Concepts, Sparse Theory, Few Data, in: Journal of European Public Policy 2/2012, S. 292-305.

19 Piotr Kaczyński: Polish Council Presidency 2011: Ambitions and Limitations, Stockholm: Swedish Institute for European Policy Studies, 2011.

20 Protokoll 14 betreffend die Euro-Gruppe, in: Amtsblatt der Europäischen Union, Nr. C 326 vom 26. Oktober 2012, S. 283.

21 The Economist: The end of the marathon?, 21.2.2012.

22 Uwe Puetter: The Eurogroup. How a secretive circle of finance ministers shape European economic governance, Manchester 2006. 
Im Zuge der Schuldenkrise sind diese bisher rein auf die Ministerebene konzentrierten Sonderstrukturen deutlich erweitert worden: Zum einen wurde mit den Eurogipfeln ein ständiges Format der Staats- und Regierungschefs der Eurostaaten geschaffen, welches nun auf oberster politischer Ebene Entscheidungen für die ,Euro-17‘ treffen kann. Ein Sondertreffen der Staats- und Regierungschefs der Eurozone wurde 2010 erstmals als besonderes Krisenforum einberufen, ist dann mit einem Beschluss des Europäischen Rates im Oktober 2011 sowie dem Fiskalpakt institutionalisiert worden. Geleitet wird das neue Gremium wie der Europäische Rat von einem ständigen Präsidenten. Als erster Amtsinhaber wurde der amtierende Präsident des Europäischen Rates Herman Van Rompuy gewählt, sodass auch in Zukunft von einer Führung der beiden Ämter in Personalunion auszugehen ist. Ein weiteres, im Zuge der Schuldenkrise geschaffenes Sonderorgan ist der Gouverneursrat des ESM, der in dem zwischenstaatlichen ESM-Vertrag die entscheidende Rolle spielt. Seine Zusammensetzung entspricht derjenigen der Eurogruppe einschließlich des Kommissars für Wirtschaft und Währung und des EZB-Präsidenten. ${ }^{23}$ Auch wurde der Präsident der Eurogruppe, JeanClaude Juncker beziehungsweise sein Nachfolger Jeroen Dijsselbloem, zum Vorsitzenden im ESM-Gouverneursrat gewählt. Zuletzt haben die Eurostaaten eine eigene Arbeitsgruppe zur Vorbereitung der Eurogruppe und der Eurogipfel eingerichtet:

\section{Abbildung 1: Die neuen Sonderstrukturen der Eurozone}

EU-27

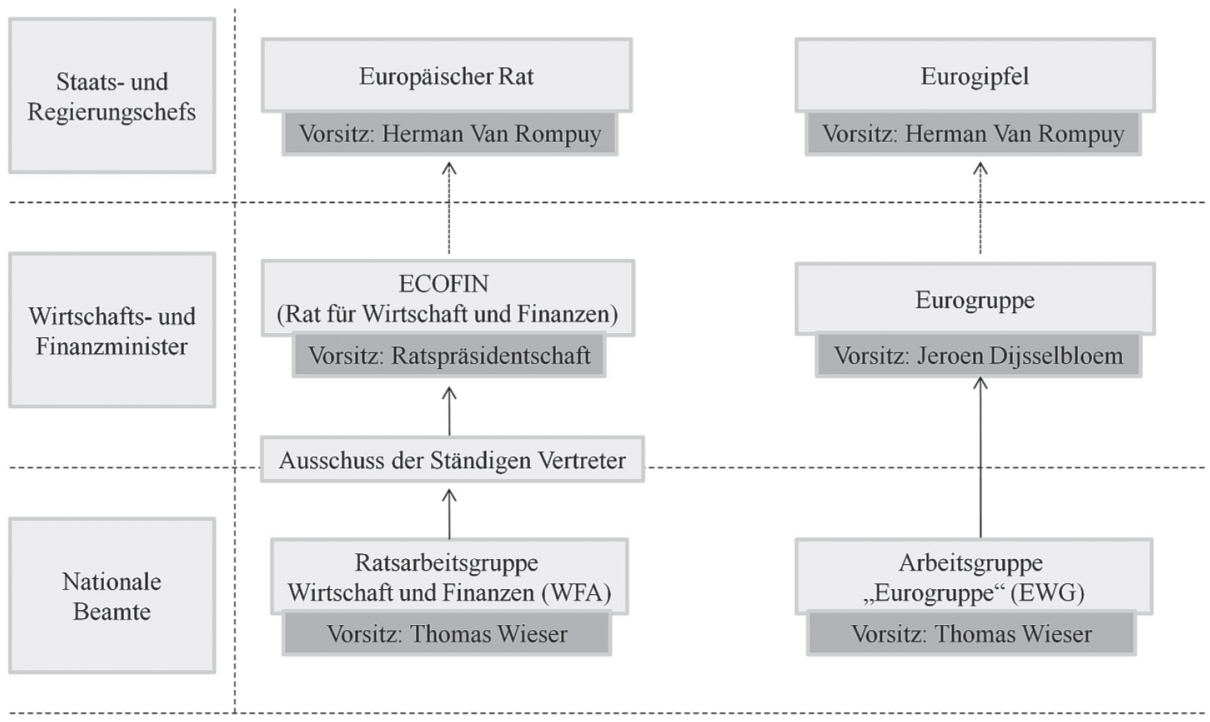

Quelle: Eigene Darstellung.

Diese Sonderstrukturen sind zwar mit den höheren Koordinationsanforderungen der Eurostaaten zu erklären. In der Praxis haben sie aber zu großen Verstimmungen zwischen den EU-Mitgliedstaaten und zu einer Verschiebung der Machtbalance in der Wirtschaftspolitik geführt. Drei Aspekte sind dabei besonders gravierend für das politische Gleichgewicht und

23 Art. 5 ESM-Vertrag. 
den politischen Zusammenhalt in der Europäischen Union: Erstens drohen die Entscheidungen, die im Rahmen der separaten Euro-Strukturen getroffen werden, auch in andere Politikbereiche der ,EU-27` überzugreifen. In Folge der europäischen Schuldenkrise waren hochpolitische Fragen der finanziellen Unterstützung einzelner Eurostaaten, die Reform der wirtschaftspolitischen Steuerung in der Europäischen Union und der allgemeinen EU-Politiken wie dem Haushalt inhaltlich kaum voneinander zu trennen. Dennoch wurden Entscheidungen, wie etwa Griechenland zusätzliche Mittel vereinfacht aus den EU-Strukturfonds zur Verfügung zu stellen, in reinen Euroformaten politisch verhandelt und die Euro-Außenseiter dann vor vollendete Tatsachen gestellt. Sollten die Eurostaaten sich wie aktuell debattiert in Wirtschaftsfragen deutlich weiter integrieren, dürften so mehr und mehr Politikbereiche entweder komplett von der Eurozone entschieden oder zumindest von den ,Euro-17` politisch vorentschieden werden.

Diese Problematik eines politischen Spill-Overs durch Vorvereinbarungen im Euro-Rahmen gefährdet besonders in Bereichen mit qualifizierter Mehrheit das Gleichgewicht in der Europäischen Union. Hier könnten die ,Euro-17` mit 213 von 255 notwendigen Stimmen nahezu alleine auch den ,EU-27` die Entscheidungen im Rat der Europäischen Union diktieren. Zudem sind die Eurogipfel aufgrund ihrer Ansiedlung auf oberster politischer Ebene prädestiniert, weiterreichende Entscheidungen in der Wirtschaftspolitik zu treffen. Diese Entwicklung wurde bei der Verhandlung des Fiskalpakts in begrenztem Maße entschärft: Angeführt von Polen konnten sich die an dem Fiskalpakt beteiligten Nicht-Eurostaaten damit durchsetzen, in Zukunft zumindest einmal jährlich an denjenigen Verhandlungen der Eurogipfel teilzunehmen, welche die Wettbewerbsfähigkeit, die institutionelle Euro-Architektur und grundlegende Fragen der Währungsunion betreffen. ${ }^{24}$

Zweitens wird durch die Konstruktion des neuen Präsidenten der Eurogipfel analog zum Amt des Präsidenten des Europäischen Rates auch die zukünftige Personalpolitik der Europäischen Union eingeschränkt. Denn beide Ämter sind de facto als Doppelhut ausgestaltet worden: Nicht nur haben die Eurostaaten mit Van Rompuy den aktuellen Präsidenten des Europäischen Rates zum Eurogipfel-Präsidenten gewählt, auch in Zukunft sollen die Amtsträger der beiden Positionen zeitgleich bestimmt werden. ${ }^{25}$ In der Folge sind beide Ämter so miteinander verknüpft, dass diese auch nach der Amtszeit von Van Rompuy in Personalunion geführt werden dürften. Für die Euro-Außenseiter ist dies doppelt relevant: Zum einen soll der Präsident der Eurogipfel nach den Vereinbarungen des Fiskalpakts die Nicht-Eurostaaten (und das Europäische Parlament) über die Ergebnisse der Sitzungen informieren und diese gegebenenfalls bei Beratungen einbeziehen. ${ }^{26}$ Zum anderen wurde damit de facto politisch festgelegt, dass in Zukunft nur Kandidatinnen und Kandidaten als Präsident des Europäischen Rates infrage kommen, die aus einem Euro-Mitgliedstaat stammen. Angesichts der hervorgehobenen politischen Bedeutung des Europäischen Rates in der Lissabonner Konstruktion eine deutliche Abwertung der Nicht-Eurostaaten in der EU-Hierarchie. ${ }^{27}$

Drittens wurde für die Vorbereitung der Sitzungen der Eurogruppe und der Eurogipfel ein administrativer Unterbau geschaffen, in dem analog zu den Arbeitsgruppen im Ratssystem Euro-Entscheidungen vorbereitet werden. Auch hier wurde de facto ein Doppelhut geschaffen: Der ständige Vorsitzende der neuen Euro-Arbeitsgruppe ist Thomas Wieser, der gleich-

24 Art. 12 Abs. 3 Fiskalpakt. Diese Möglichkeit wurde jedoch erst eingefügt, nachdem Polen und Schweden gedroht hatten, den Vertrag nicht zu unterzeichnen, falls sie nicht an den Eurogipfeln teilnehmen können.

25 Euro-Gipfel: Erklärung des Euro-Gipfels. 26. Oktober 2011.

26 Art. 12 Abs. 5-6 Fiskalpakt.

27 Philippe de Schoutheete: The European Council and the Community Method. Notre Europe Policy Paper $56 / 2012$. 
zeitig die Ratsarbeitsgruppe Wirtschaft und Finanzen leitet. ${ }^{28}$ Obgleich diese Arbeitsgruppen auf den ersten Blick nur Vorbereitungsarbeit leisten sollen, ist der langfristige Effekt dieser Entscheidung nicht zu unterschätzen: Im regulären EU-System spielen die vorbereitenden Ausschüsse im Ratssystem eine fundamentale Rolle für die Entscheidungsfindung und vereinbaren Studien zufolge einen Großteil der späteren Entscheidungen im Rat soweit, dass sie von den Ministern oft nur noch formell akzeptiert werden. ${ }^{29}$ Hinzukommt, dass die EuroArbeitsgruppe anders als reguläre Ratsarbeitsgruppen nicht an den Ausschuss der Ständigen Vertreter berichtet, in dem alle Mitgliedstaaten vertreten sind, sondern direkt an die exklusive Eurogruppe.

Etwas abgeschwächt wird diese Tendenz zur engeren Zusammenarbeit der ,Euro-17` in den neuen Sonderinstitutionen nur durch die Teilnahme der Präsidenten des Europäischen Parlaments und der EZB an den Eurogipfeln. Politisch ist deren Rolle jedoch höchst unterschiedlich: Auf der einen Seite ist die Teilnahme des Präsidenten des Europäischen Parlaments (noch) vornehmlich als symbolisch einzuschätzen. Wie auch bei den regulären Treffen des Europäischen Rates gab es bei den vergangenen Eurogipfeln relativ zu Beginn der Sitzung einen formellen Austausch mit ihm, der Zutritt zu den eigentlichen Verhandlungen blieb dem Parlamentspräsidenten aber verwehrt. Langfristig bietet sich jedoch auch für das Parlament eine Möglichkeit, über diesen Weg auf die Verhandlungen Einfluss zu nehmen, besonders dann wenn die Zustimmung des Parlaments für einzelne Entscheidungen erforderlich wird. Im Gegensatz dazu hat der EZB-Präsident auch bei den Verhandlungen der Eurogipfel in dem Maße an Bedeutung gewonnen, wie auch die EZB für die Eindämmung der Schuldenkrise wichtiger wurde. ${ }^{30}$

Mit dem Ausbau der administrativen Kapazitäten ist also in der Euro-Architektur eine vertikal durchgängige Trennung vollzogen worden (siehe Abbildung 1), die auf allen Ebenen die Nicht-Eurostaaten ausschließt und de facto eine neue, unabhängige Säule in der Ratsstruktur bildet. Für die Nicht-Eurostaaten ist dies eine gefährliche Situation: Zwar können sie sich über den Euro-Plus-Pakt an Beratungen der Eurostaaten über wirtschaftspolitische Koordinierung beteiligen und haben sich durch den Fiskalpakt zumindest in begrenztem Rahmen eine Beteiligung an den Eurogipfeln erstritten. In der Gesamtheit aber ist ein System auf den Weg gebracht worden, dass den Eurostaaten weitreichende Räume bietet und systematisch Anreize schafft, um im erweiterten Rahmen der informellen Euro-Architektur zumindest politisch die Entscheidungen für die Wirtschaftspolitik der Union zu treffen. Diese enge und vor allem vertrauliche Abstimmung ist angesichts der politischen Sensibilität und Bedeutung der Eurozonen-Entscheidungen funktional notwendig geworden. Dennoch gilt, umso mehr wichtige Entscheidungen nur zwischen den Eurostaaten in den Eurogremien getroffen werden, umso mehr entwickelt sich die Eurozone zu einer Union in der Union.

\section{Verschiebung der politischen Gewichte in und zum Rat}

Doch auch innerhalb der Eurogruppe und anderen Formen differenzierter Integration verschieben sich die politischen Gewichte durch die veränderte Zusammensetzung von Mit-

28 Economic and Financial Committee: EU Economic and Financial Committee: President, abrufbar unter: http:// europa.eu/efc/president/index_en.htm (Zugriff: 7.11.2012).

29 Frank Häge: Who decides in the Council of the European Union, in: Journal of Common Market Studies 3/2008, S. 533-558.

30 Zur Rolle der EZB in der Krise und der Reform der Eurozone, siehe Francesco Drudi/Alain Durre/Francesco Paolo Mongelli: The Interplay of Economic Reform and Monetary Policy: The Case of the Eurozone, in: Journal of Common Market Studies 6/2012, S. 881-898. 
gliedstaaten. Es gehört zu den wenig umstrittenen Feststellungen über die Auswirkungen der Schuldenkrise, dass sich das Gewicht Deutschlands in der Europäischen Union aufgrund seiner Schlüsselrolle im Euro-Krisenmanagement drastisch vergrößert hat. Diese neue hervorgehobene Stellung wird in der Regel primär auf die aktuelle wirtschaftliche Stärke Deutschlands zurückgeführt. Entscheidende Impulse - und Vetos - zu den Rettungspaketen für Eurostaaten sowie die Weiterentwicklung der institutionellen Architektur der Eurozone kamen aus Berlin. In vielen Bereichen konnte sich Deutschland, oft in Abstimmung mit Frankreich, durchsetzen, etwa bei der Vertragsänderung zur Einführung des ESM, der Ausgestaltung des Fiskalpakts oder der bisherigen Ablehnung von Eurobonds. ${ }^{31}$

Aber auch die Mechanismen der differenzierten Integration verschieben - innerhalb und außerhalb des Krisenmoments - die politischen Gewichte in der Union. Das Ausmaß und die Richtung der Veränderung richten sich primär nach drei Faktoren: Erstens verschiebt sich durch veränderte Abstimmungsmodi rein arithmetisch das Stimmengewicht der einzelnen Mitgliedstaaten im Rat der Europäischen Union. So sieht der EU-Vertrag bei der Verstärkten Zusammenarbeit ebenso wie bei anderen Formen der differenzierten Integration vor, dass nach denselben Abstimmungsregeln entschieden wird wie bei den ,EU-27 '. ${ }^{32} \mathrm{Kommt}$ die qualifizierte Mehrheit zur Anwendung wird demnach das erforderliche Quorum für eine Zustimmung in Relation zum Quorum bei Beteiligung aller Mitgliedstaaten gebildet, welches knapp 74 Prozent der Stimmen entspricht. ${ }^{33}$ Auf den ersten Blick scheint diese Regelung die Stimmenverteilung zwischen den beteiligten Staaten aufrechtzuerhalten, beispielsweise behält Deutschland ebenso wie Frankreich 29 Stimmen. Bei genauerer Betrachtung aber verändert der Wegfall vor allem größerer Staaten das relative Gewicht der Staaten untereinander und im Vergleich zum Quorum für die qualifizierte Mehrheit sowie die Sperrminorität. Wie in Tabelle 1 ersichtlich ist dieser Effekt umso stärker, je weniger Mitgliedstaaten sich an einem Projekt der differenzierten Integration beteiligen.

Die praktischen Auswirkungen dieser Verschiebung lassen sich sehr eindrucksvoll am Beispiel der Eurozone illustrieren. Wenn der Rat der Europäischen Union Beschlüsse zur haushaltspolitischen Überwachung trifft, so haben gemäß Art. 136 Abs. 2 EUV nur die EuroMitgliedstaaten Stimmrecht. Die Gesamtstimmenanzahl der 17 Eurostaaten beträgt 213 Stimmen, sodass für eine qualifizierte Mehrheit 158 Stimmen notwendig sind. In der Folge entstehen zum einen neue Sperrminoritäten, da für die dauerhafte Blockade einer Entscheidung nun 56 Stimmen ausreichen. Deutschland und Frankreich, die zusammen 58 Stimmen stellen, können hier also jeden Beschluss blockieren. Diese Blockademacht verleiht ihnen neben ihrer wirtschaftlichen Stärke zusätzliches politisches Gewicht in der Eurozone.

31 William E. Paterson: The Reluctant Hegemon? Germany Moves Centre Stage in the European Union, in: Journal of Common Market Studies S1/2011, S. 57-75.

32 Art. 238 Abs. 3 AEUV. In Verbindung mit dem Protokoll 36 über die Übergangsbestimmungen gelten bis 2014 noch die gewogenen Stimmrechte, wie sie im Vertrag von Nizza festgelegt wurden. Anschließend gelten dieselben prozentualen Anteile (55 Prozent der beteiligten Staaten, die 65 Prozent der Bevölkerung der beteiligten Staaten repräsentieren) wie bei Abstimmungen mit allen Mitgliedstaaten.

33 Dies entspricht den 255 Stimmen, die bei den Gesamtstimmen von 345 für die qualifizierte Mehrheit notwendig sind, also 73,913 Prozent. 
Tabelle 1: Veränderung der Gesamtstimmen und der qualifizierten Mehrheit im Rat

\begin{tabular}{|c|c|c|c|c|}
\hline & Staaten & $\begin{array}{l}\text { Gesamt- } \\
\text { stimmen }\end{array}$ & $\begin{array}{l}\text { Qualifizierte } \\
\text { Mehrheit }\end{array}$ & Sperrminorität \\
\hline EU & 27 & 345 & 255 & 91 \\
\hline GSVP & 26 & Einstimmig & - & Vetorecht \\
\hline EU-Patent & 25 & 289 & 214 & 76 \\
\hline $\mathrm{RFSR}^{34}$ & 24 & 302 & 224 & 79 \\
\hline Euro & 17 & 213 & 158 & 56 \\
\hline $\begin{array}{l}\text { Transnationales } \\
\text { Scheidungsrecht }\end{array}$ & 15 & 199 & $(147)^{35}$ & (53) \\
\hline Finanztransaktionssteuer & 11 & 175 & $(130)^{36}$ & $(46)$ \\
\hline
\end{tabular}

Quelle: Schriftliche Nachfrage, 2012, Generalsekretariat des Rates der Europäischen Union.

Zum anderen steigt das relative Stimmengewicht der beteiligten Staaten, wovon diejenigen mit hohen Stimmrechten am stärksten profitieren. Deutschland, das aktuell gemeinsam mit Frankreich, Großbritannien und Italien beziehungsweise ab 2014 aufgrund seiner Bevölkerungsgröße alleine über die meisten Stimmen verfügt, gewinnt also am stärksten bei dieser Verschiebung. So lassen sich auf Grundlage des Stimmengewichts und des Mehrheitsquorums die Veränderungen in der Abstimmungsmacht der Mitgliedstaaten berechnen. Hierfür wurde ein anerkannter Machtindex genutzt, welcher auf Grundlage der Stimmrechte die Abstimmungsmacht eines Akteurs im Spektrum zwischen 1 - der Akteur kann jede Entscheidung bestimmen - und 0 für einen Akteur ohne Einfluss berechnet (siehe Tabelle 2).

Ein solcher Machtindex stellt selbstverständlich nur eine mathematische Annäherung an politische Macht dar, welche in der politischen Praxis neben dem Stimmrecht von weiteren, zum Teil situativen Faktoren wie Wirtschaftskraft, Handlungsressourcen in dem jeweiligen Politikfeld, innenpolitische Stärke etc. beeinflusst wird. Doch auch hier greift - selbst bei Einstimmigkeit - grundsätzlich dasselbe mathematische Prinzip: Sinkt die Anzahl der Mitglieder, steigt das individuelle Gewicht der verbleibenden Staaten mit überproportionalem Einflussgewinn der großen Mitglieder.

Ein zweiter wichtiger politischer Faktor für die Richtung der Verschiebung politischen Einflusses durch differenzierte Integration ist die veränderte Verteilung von politischen Präferenzen der Mitgliedstaaten. So wird das Voranschreiten einer Gruppe von Mitgliedstaaten in den seltensten Fällen das Spektrum an Interessen in der ,EU-27` identisch abbilden. Im Gegenteil, in der Regel sollten diejenigen Mitgliedstaaten enger zusammenarbeiten, die zu-

34 Unter der Annahme, dass die drei Opt-Out-Staaten - Dänemark, Großbritannien, Irland - nicht von ihrem OptIn-Recht Gebrauch machen. Bei Gebrauch zählen sie wieder zu den beteiligten Mitgliedstaaten mit entsprechenden Folgen für die Gesamtstimmen beziehungsweise das Mehrheitsquorum.

35 Die Verstärkte Zusammenarbeit zur Einführung eines transnationalen Scheidungsrechts beruht auf Art. 81 Abs. 3 AEUV, der ein besonderes Gesetzgebungsverfahren mit Einstimmigkeit im Rat vorsieht. Rechtlich wäre aber im Rahmen der Verstärkten Zusammenarbeit gemäß Art. 333 AEUV eine Überführung in das ordentliche Gesetzgebungsverfahren mit qualifizierter Mehrheit möglich.

36 Die Einführung der Finanzmarkttransaktionssteuer wird nach Vorschlag der Kommission auf Art. 113 AEUV beruhen. Demnach könnten Entscheidungen in diesem Bereich nur einstimmig beschlossen werden, obgleich rechtlich wie auch bei dem Scheidungsrecht eine Überführung ins ordentliche Gesetzgebungsverfahren möglich wäre. Vgl. Europäische Kommission: Vorschlag für einen Beschluss des Rates, 2012. 
mindest in einem begrenzten Rahmen politische Interessen teilen. In der Folge können Positionen von Mitgliedstaaten übergangen werden, während sich die Mehrheitspräferenzen deutlich verschieben können. Ein Beispiel für den ersten Fall ist das europäische Patentrecht, in dem die Präferenzen Italiens und Spaniens übergangen werden konnten. Diese reagierten darauf mit einer Klage vor dem Europäischen Gerichtshof. ${ }^{37}$

Tabelle 2: Verschiebung der Abstimmungsmacht ausgewählter Mitgliedstaaten ${ }^{38}$

\begin{tabular}{|l|c|c|c|}
\hline Mitgliedstaat & $\begin{array}{c}\text { Stimmgewicht } \\
\text { im Rat }\end{array}$ & $\begin{array}{c}\text { Machtindex } \\
\text {,EU-27 }\end{array}$ & $\begin{array}{c}\text { Machtindex } \\
\text { Euro-17 }^{\mathbf{6}}\end{array}$ \\
\hline Deutschland & 29 & 0,087 & 0,149 \\
\hline Frankreich & 29 & 0,087 & 0,149 \\
\hline Spanien & 27 & 0,079 & 0,141 \\
\hline Niederlande & 13 & 0,037 & 0,060 \\
\hline Österreich & 10 & 0,028 & 0,043 \\
\hline Finnland & 7 & 0,020 & 0,029 \\
\hline Luxemburg & 4 & 0,011 & 0,017 \\
\hline
\end{tabular}

Quelle: Eigene Berechnung.

Folgenreich auch für beteiligte Mitgliedstaaten kann die Verschiebung der Mehrheitsposition innerhalb eines Projekts differenzierter Integration sein. Unter der Annahme, dass Entscheidungen im Rat der Europäischen Union in der Regel einen Kompromiss zwischen den Interessen aller Mitgliedstaaten darstellen, können durch das Wegfallen einzelner Staaten wichtige politische Verbündete verloren gehen. So ist es aus deutscher Sicht nicht unproblematisch, wenn in Zukunft vermehrt wirtschaftspolitische Entscheidungen im Euroformat getroffen werden. EU-Mitgliedstaaten mit ausgeprägter Stabilitätskultur wie Polen und Schweden oder größeren Übereinstimmungen in wirtschaftspolitischen Grundinteressen wie Großbritannien stehen dann nicht als Bündnispartner zur Verfügung. ${ }^{39}$

Drittens ist die differenzierte Integration rund um die Eurozone in ihrer aktuellen Ausgestaltung primär intergouvernementaler Natur: Anstatt über die supranational ausgestaltete Verstärkte Zusammenarbeit oder vertraglich festgelegte Opt-Outs zu gehen, haben die ,Euro-17 ' und die beteiligten Pre-Ins mit dem Euro-Plus-Pakt auf intergouvernementale Koordinierung und mit dem Fiskalpakt auf einen völkerrechtlichen Nebenvertrag gesetzt. Damit läuft der Großteil der Entscheidungen in der differenzierten Integration rund um die Eurozone am ordentlichen Gesetzgebungsverfahren vorbei. In der Folge hat sich die komplette dargestellte Sonderstruktur der Eurozone auf das Ratssystem konzentriert, in dem die wesentlichen

37 Rat der Europäischen Union: Case before the Court of Justice of the European Union - Case C-274/11(Kingdom of Spain against Council of the European Union) - Enhanced cooperation in the area of the creation of unitary patent protection, Dok. 12500/11.

38 Für die Berechnung der Abstimmungsmacht wird der Shapley-Shubik-Index (SSI) genutzt, ein anerkannter Machtindex. Der SSI beruht auf dem Grundgedanken, dass ein Akteur umso mehr Abstimmungsmacht besitzt, je häufiger seine Stimme für eine Koalition entscheidend ist, um das notwendige Quorum für einen Beschluss zu erreichen. Für eine Anwendung des SSI auf Verhandlungen in der Europäischen Union siehe Robert Thomson/Frans N. Stokman: Research design: measuring actors' positions, saliences and capabilities, in: Robert Thomson u.a. (Hrsg.): The European Union decides, Cambridge 2006, S. 25-53.

39 Jean Pisani-Ferry: Only One Bed for Two Dreams: A Critical Retrospective on the Debate over the Economic Governance of the Euro Area, in: Journal of Common Market Studies 4/2006, S. 823-844. 
politischen Entscheidungen für die gemeinsame Währung getroffen werden. Das institutionelle Gleichgewicht der Europäischen Union wird so in der Schuldenkrise vorerst zum Rat der Europäischen Union und dabei vor allem zu den Staats- und Regierungschefs im Europäischen Rat beziehungsweise den Eurogipfeln verschoben.

\section{Die Kommission als Garant des Unionsinteresses}

Anders stellt sich das Problem für die Kommission dar. Als Vertreterin des gemeinschaftlichen Interesses kann und hat die Kommission ihre Aufgabenbereiche bis dato nicht nach der differenzierten Integration aufgeteilt. Entscheidungen etwa zur Innen- und Justizpolitik oder zur Eurozone werden wie andere Entscheidungen auch vom Kollegium der Kommission im Konsens getroffen. Kommissare, die weder rechtlich noch politisch Vertreter ihrer Staaten sind, haben dabei unabhängig vom Status ihrer Herkunftsstaaten das gleiche Stimmrecht.

Auch auf dem Level der normalen Kommissionsbeamten wirkt sich die Differenzierung kaum aus. Im Vergleich zum Anteil an der gesamten Belegschaft der Kommission sind etwa britische, dänische und irische Beamte in den Generaldirektionen mit Bezug zum RFSR nur unwesentlich schwächer repräsentiert als in anderen Generaldirektionen. ${ }^{40}$ Bei der Besetzung der direkten Entscheidungspositionen hingegen existiert informell eine klare Trennung - seit Einführung der Opt-Outs aus der gemeinsamen Währung und dem RFSR sowie Schengen wurde keiner der zuständigen Kommissarsposten mit einer Kandidatin oder einem Kandidaten aus den nicht-beteiligten Staaten besetzt. ${ }^{41}$ Darüber hinaus wurden im selben Zeitraum auch nur Politiker zum Kommissionspräsidenten gewählt, die aus Staaten stammen, die sich an allen Projekten differenzierter Integration beteiligen. Auch in der öffentlichen Debatte wurde explizit darauf verwiesen, dass ein Kommissionspräsident die Interessen der wichtigsten Differenzierungsprojekte, der gemeinsamen Währung, dem Schengenraum und dem RFSR gegenüber den Unionsbürgerinnen und -bürgern, den anderen EU-Institutionen sowie nach außen vertreten müsse.

Nicht zuletzt kommt der Kommission als Hüterin der Verträge eine hervorgehobene Rolle als dasjenige Organ zu, welches den Zusammenhalt der Union sichern soll. Demnach soll sie bei einer Verstärkten Zusammenarbeit sicherstellen, dass diese mit dem Recht der Europäischen Union vereinbar ist, und als Transmissionsriemen die nicht-beteiligten Mitgliedstaaten sowie das Europäische Parlament unterrichten. Sie wird damit für die nicht-beteiligten Mitglieder zum zentralen Ansprechpartner und für die beteiligten Mitglieder zum Garanten europäischer Legitimität. Solange die Differenzierung also im EU-Rahmen durchgeführt wird, kann die Kommission sogar gestärkt aus dieser Entwicklung herausgehen.

\section{Das demokratisch-legitimatorische Dilemma des Europäischen Parlaments}

Am kritischsten aus institutioneller Sicht ist die differenzierte Integration für das Europäische Parlament. Zunächst ist zwar festzustellen, dass eine Beteiligung des Parlaments an Entscheidungsverfahren von Projekten differenzierter Integration keineswegs ausgeschlossen ist, solange sie im EU-Rahmen angesiedelt sind. In der Praxis stellt sie abseits der neuen Euro-Strukturen sogar die Norm dar - bei von Opt-Outs betroffenen Bereichen ist das Par-

40 Dies sind im engeren Sinne die Generaldirektionen für Justiz (JUST) und Inneres (HOME). Im März 2012 waren in beiden Generaldirektionen Mitarbeiter aus allen drei Opt-Out-Staaten vertreten. Vgl. Europäische Kommission: Distribution of officials and temporary agents by Directorate General and nationality (all budgets). March 2012, abrufbar unter: http://ec.europa.eu/civil_service/docs/europa_sp2_bs_nat_x_dg_en.pdf (letzter Zugriff: 19.3.2012).

41 Eigene Erhebung auf Basis offizieller Informationen der Europäischen Union. 
lament als Ganzes wie im jeweiligen Politikbereich üblich beteiligt, das heißt beispielsweise nach dem Vertrag von Lissabon mit vollem Mitentscheidungsrecht in der Innen- und Justizpolitik. Kommt also im RFSR das ordentliche Gesetzgebungsverfahren zur Anwendung, ist das Europäische Parlament auch bei Rechtsakten, aus denen einzelne Mitgliedstaaten OptOuts haben, regulär und vollständig als Mitgesetzgeber beteiligt. Ebenso nimmt das Parlament in der Gesetzgebung im Bereich der wirtschaftspolitischen Steuerung mit besonderem Bezug zur Eurozone seine üblichen Funktionen wahr.

Deutlichstes Beispiel für eine volle Beteiligung des Parlaments waren die Beschlüsse zur Reform des Stabilitäts- und Wachstumspakts in Form des sogenannten Six-Packs. ${ }^{42}$ Hier konnte sich das Parlament mit weitreichenden Forderungen gegenüber den Mitgliedstaaten durchsetzen und eine Verschärfung in einigen Punkten erreichen. ${ }^{43}$ Nicht zuletzt ist die Einrichtung einer Verstärkten Zusammenarbeit nur nach Zustimmung des Parlaments möglich, in der dann in der Regel nach dem ordentlichen Gesetzgebungsverfahren mit vollem Mitentscheidungsrecht entschieden wird. Den bisher beschlossenen Rechtsakten unter Anwendung der Verstärkten Zusammenarbeit beim EU-Patent, der Regelung transeuropäischer Scheidungen und der Finanztransaktionssteuer, hat also jeweils das Parlament zugestimmt.

Mit der vollen Beteiligung an Entscheidungsverfahren innerhalb der EU-Strukturen beginnt aber eigentlich erst das politisch-legitimatorische Dilemma, dem das Europäische Parlament durch die differenzierte Integration ausgesetzt ist: Die Grundidee der differenzierten Integration als Trennung der Mitglieder einer Gemeinschaft in Beteiligte und Nicht-Beteiligte erfordert konsequenterweise auch eine Differenzierung in den damit verbundenen Entscheidungsverfahren. Wie dargestellt ist es im Rat der Europäischen Union vollkommen selbstverständlich, dass Vertreter von Opt-Out-Staaten auch kein Stimmrecht bei Entscheidungen in diesen Bereichen besitzen. Eine äquivalente Vorgehensweise würde vom Europäischen Parlament allerdings verlangen, dass etwa Abgeordnete aus Großbritannien in Fragen der gemeinsamen Währung nicht abstimmen dürften und das Parlament de facto je nach Entscheidung unterschiedlich zusammengesetzt sein müsste. Dies wird im Parlament bisher mehrheitlich deutlich abgelehnt. So betonte die Konferenz der Präsidenten des Europäischen Parlaments im Oktober 2012: „Das Parlament des Euro ist das Europa-Parlament.“44

Bisher ist das Parlament daher sowohl bei der Verstärkten Zusammenarbeit als auch bei anderen bisherigen Formen differenzierter Integration im EU-Rahmen wie Opt-Outs oder dem Schengener Abkommen in seiner Gesamtheit mit allen Abgeordneten beteiligt. Diese Praxis ist aber aus Sicht demokratischer Legitimation problematisch, denn sie gibt Parlamentariern ein Mitspracherecht, deren Mitgliedstaaten und Wahlkreise eigentlich nicht von der jeweiligen Entscheidung betroffen sind. Im Zweifelsfall können diese Parlamentarier das Votum des Europäischen Parlaments entscheidend verändern, in dem sie die Stimmenverteilung in der Versammlung verschieben. Wenn das Parlament in Fragen der Währungsunion abstimmt, etwa in seinen Resolutionen zur Eurokrise, sind aktuell 273 Abgeordnete (36,2 Prozent des Parlaments) stimmberechtigt, obwohl ihre Mitgliedstaaten nicht dem Euro angehören. Sollte die geplante Verstärkte Zusammenarbeit in der Finanzmarkttransaktions-

42 Obgleich der Stabilitäts- und Wachstumspakt primär auf die Mitgliedstaaten der Eurozone abzielt, entfaltet er auch eine begrenzte Wirkung auf die Nicht-Eurostaaten. Formal wurden die sechs Gesetzgebungsinitiativen des Six-Packs daher auch von der Europäischen Union insgesamt verabschiedet.

43 Für eine detaillierte Analyse der Reformen der wirtschaftspolitischen Steuerung und des Stabilitäts- und Wachstumspakts mit dem Six-Pack siehe Stijn Verhelst: The reform of European economic governance: Towards a sustainable monetary union?, Egmont Paper 47/2011.

44 Benjamin Fox: No eurozone-only assembly, say MEPs, in: EUobserver, 6.10.2012 (eigene Übersetzung). 
steuer in das ordentliche Gesetzgebungsverfahren überführt werden, ${ }^{45}$ würden sogar über 45 Prozent Abgeordnete mitbestimmen, die Wahlkreise aus nicht beteiligten EU-Mitgliedstaaten vertreten:

Tabelle 3: Das Europäische Parlament und die differenzierte Integration

\begin{tabular}{|l|c|c|c|}
\hline & $\begin{array}{c}\text { MdEP aus } \\
\text { beteiligten Staaten }\end{array}$ & $\begin{array}{c}\text { MdEP aus nicht- } \\
\text { beteiligten Staaten }\end{array}$ & $\begin{array}{c}\text { Stimmanteil MdEP } \\
\text { aus nicht-beteilig- } \\
\text { ten Staaten }\end{array}$ \\
\hline EU & 754 & 0 & $0 \%$ \\
\hline GSVP & 741 & 13 & $1,7 \%$ \\
\hline EU-Patent & 627 & 127 & $16,9 \%$ \\
\hline RFSR 46 & 656 & 98 & $13,0 \%$ \\
\hline Euro & 481 & 273 & $36,2 \%$ \\
\hline $\begin{array}{l}\text { Transnationales } \\
\text { Scheidungsrecht }\end{array}$ & 465 & 289 & $38,3 \%$ \\
\hline $\begin{array}{l}\text { Finanztransaktions- } \\
\text { steuer }\end{array}$ & 412 & 342 & $45,3 \%$ \\
\hline
\end{tabular}

Quelle: Eigene Berechnung.

Während diese Problematik bei Bereichen differenzierter Integration mit nur wenigen Nicht-Teilnehmern wie der GSVP, dem RFSR oder dem EU-Patent nur begrenzte Bedeutung hat, wird die demokratische Legitimität des Europäischen Parlaments in Euro-Fragen dadurch fundamental untergraben. Bis dato hat das Parlament versucht vor allem auf informellem Wege auf diese Herausforderung zu reagieren. In der Regel werden Abgeordnete aus einem Mitgliedstaat, welcher sich an einem Projekt nicht beteiligt, auch nicht zum Berichterstatter mit Bezug auf das jeweilige Projekt ernannt - eine in den Verhandlungen mit dem Rat und der Kommission sehr einflussreiche Position. ${ }^{47}$ So wurde in den letzten beiden Legislaturperioden kein dänischer Abgeordneter als Berichterstatter mit Fragen der GSVP betraut. ${ }^{48}$ Ebenso stammten bis auf in zwei Ausnahmen ${ }^{49}$ alle Berichterstatter zu Entscheidungen mit direktem Bezug zur gemeinsamen Währung aus Staaten, die bereits den Euro eingeführt haben. Auch in der Innen- und Justizpolitik ist diese Praxis zu beobachten. Hier waren während der letzten beiden Legislaturperioden keine Abgeordneten der Opt-OutStaaten Dänemark oder Irland Berichterstatter zum RFSR. Das Gewicht der britischen Ab-

45 Dies ist im bisherigen Vorschlag der Kommission nicht vorgesehen, aber nach Art. 333 AEUV rechtlich möglich.

46 Trotz des Opt-In-Rechts werden die drei Opt-Out Staaten - Dänemark, Großbritannien, Irland - hier als komplette Außenseiter gewertet. Eine Anpassung des Europäischen Parlaments an differenzierte Integration wäre aufgrund dieser Opt-In-Rechte mit zusätzlichen Herausforderungen konfrontiert.

47 Zur politischen Bedeutung der Berichterstatter im Europäischen Parlament, siehe Richard Corbett/Francis Jacobs: The European Parliament, 8. Auflage, London 2011.

48 Eigene Erhebung auf Grundlage offizieller Informationen des Europäischen Parlaments. Untersucht wurden die Legislaturperioden 2004-2009 sowie 2009-2012. Siehe Legislative Observatory of the European Parliament, abrufbar unter: http://www.europarl.europa.eu/oeil/home/home.do?lang=en (letzter Zugriff: 3.2.2012).

49 Diese Ausnahmen waren ein Bericht zur Bekämpfung von Eurofälschung durch den bulgarischen Abgeordneten Slavi Binev sowie ein Bericht der britischen Abgeordneten Vicky Ford zur Reform des Stabilitäts- und Wachstumspakts. 
geordneten hingegen scheint für diese ,natürliche Selektion` zu groß zu sein - so wurden 36 der insgesamt 170 Dossiers zum RFSR von britischen Mitgliedern des Europäischen Parlaments betreut. ${ }^{50}$ Angesichts der weitreichenden Opt-Outs Großbritanniens stellt sich hier die Frage, inwieweit eine solche hervorgehobene Stellung politisch zu legitimieren ist.

Tabelle 4: Berichterstatter im Europäischen Parlament zu Projekten differenzierter Integration (2009-2011)

\begin{tabular}{|l|c|c|c|}
\hline & $\begin{array}{c}\text { Berichterstatter } \\
\text { aus beteiligten } \\
\text { Staaten }\end{array}$ & $\begin{array}{c}\text { Berichterstatter } \\
\text { aus nicht-beteilig- } \\
\text { ten Staaten }\end{array}$ & Gesamt \\
\hline RFSR & 139 & 31 (nur VK) & 170 \\
\hline Euro & 19 & 2 (VK/Bulgarien) & 21 \\
\hline
\end{tabular}

Quelle: Eigene Berechnung auf Basis von Angaben des Europäischen Parlaments.

Insgesamt hat das Europäische Parlament mit dieser Strategie lange eine politische Debatte über seine Zusammensetzung in Fragen differenzierter Integration vermieden. Mit der Intensivierung der Zusammenarbeit in der Eurozone und den damit verbundenen Doppelstrukturen im Rat der Europäischen Union wird sich aber auch für die parlamentarische Seite der EU-Organe die Frage stellen, wie eine demokratisch-legitimierte Beteiligung an Entscheidungen gewährleistet werden kann.

Einen einfachen Ausweg aus diesem Dilemma scheint es nicht zu geben: Bislang besteht das Parlament auf seiner Integrität und auf eine Beteiligung aller Parlamentarier auch in Eurofragen. Damit gefährdet es aber seinen Anspruch auf demokratische Legitimität, wenn zum Teil mehr als ein Drittel der Stimmen von Vertretern eigentlich nicht betroffener Bevölkerungen stammen. Diese Argumentation unterminiert das Gewicht des Parlaments und seine Forderung nach mehr Beteiligung zur Stärkung der demokratischen Legitimation. Zusammen mit der Verschiebung hin zum Rat der Europäischen Union wird es so zu einem Verlierer der differenzierten Integration.

In der politischen Debatte gewinnen daher zunehmend Vorschläge an Bedeutung, die eine Stärkung der parlamentarischen Mitbestimmung in Fragen der Wirtschafts- und Währungsunion über eine institutionelle Reform erreichen wollen. Die Vorschläge gehen vor allem in zwei Richtungen. Auf der einen Seite des Spektrums steht die Idee, dass in Eurofragen nur Abgeordnete aus Euro-Mitgliedstaaten abstimmen dürften. Ein solches Abrücken von der Einheitlichkeit des Parlaments und eine Differenzierung der Stimmrechte nach Herkunftsstaaten widersprechen aber nicht nur der Idee des Parlaments als supranationales Organ, in dem übergreifend die Bürger der Union vertreten werden. Vor allem sind sie auch für die Arbeit des Parlaments und seiner Fraktionen von höchster politischer Sprengkraft. Parlamentarier aus Nicht-Eurostaaten liefen dann Gefahr, zu Abgeordneten zweiter Klasse zu werden. Auf der anderen Seite schlagen sowohl der Quadriga-Bericht zur Reform der Wirtschafts- und Währungsunion von Dezember $2012^{51}$ als auch vermehrt nationale Parlamentarier die Schaffung eines neuen, separaten parlamentarischen Gremiums vor, in dem sich Vertreter der nationalen Parlamente und des Europäischen Parlaments zur Eurozone aus-

50 Eigene Erhebung. Untersucht wurden nur die beiden direkt mit Fragen des RFSR betrauten Ausschüsse, also diejenigen für Bürgerliche Freiheiten, Justiz und Inneres (LIBE) sowie der Rechtsausschuss (JURI).

51 Europäischer Rat: Auf dem Weg zu einer echten Wirtschafts- und Währungsunion, 2012. 
tauschen können. Damit wäre ein solches Gremium aber auf den reinen Informationsaustausch beschränkt.

Konfrontiert mit diesen politisch schwierigen Optionen wird auch das Parlament bei der kommenden Reform eine Balance zwischen Erhaltung der gemeinsamen Integrität aller EUMitgliedstaaten sowie Handlungsfähigkeit und demokratischer Legitimation für die Entscheidungen der Eurozone finden müssen.

\section{Eine Spaltung im System}

Seit dem Vertrag von Maastricht funktioniert die Europäische Union mit einem gewissen Grad an differenzierter Integration. Die Analyse der konkreten Auswirkungen auf die institutionellen Strukturen der Union hat aufgezeigt, dass die Integrität der Zusammenarbeit unter 27 und der einzelnen EU-Institutionen dabei aufrecht erhalten wurde. Hierzu hat zum einen beigetragen, dass die Außenseiter über den gemeinsamen institutionellen Rahmen der Europäischen Union zumindest in begrenztem Maße über die Verhandlungen und Entscheidungen in den jeweiligen Projekten informiert und beteiligt waren. Im modularen Rat der Europäischen Union konnten sie als Beobachter ohne Stimmrecht an den Verhandlungen teilnehmen, während das Europäische Parlament und die Kommission in ihrer Gänze beteiligt waren. Bis 2010 wäre dies das positive Fazit der Analyse gewesen.

Mit der Ausweitung der differenzierten Integration während der Schuldenkrise hat aber eine Dynamik ihren Lauf genommen, mit der die Europäische Union in drei separate, zunehmend auch institutionell voneinander getrennte Gruppen geteilt wird: Die ,Euro-17`, die Pre-Ins und die dauerhaften Außenseiter. Am deutlichsten ausgeprägt ist diese Trennung im Ratssystem der Europäischen Union, in dem eine vollständig separate institutionelle Struktur für Angelegenheiten der gemeinsamen Währung geschaffen wurde. Mit Euro-Gipfeln, EuroGruppe und Euro-Arbeitsgruppe sowie dem ESM-Gouverneursrat haben die ,Euro-17` auf allen Ebenen exklusive Foren für ihre Beratungen geschaffen. Auch die Pre-Ins werden hierbei nur begrenzt eingebunden, haben aber über den Euro-Plus-Pakt und den Fiskalpakt zumindest Informationsrechte und eine regelmäßige Teilnahme an den Eurogipfeln erreicht. Hinzukommt, dass die während der Schuldenkrise eingeführten Reformen auch das institutionelle Gleichgewicht vorerst zum Ratssystem vor allem in Richtung Europäischen Rat beziehungsweise Eurogipfel verschoben haben. Obgleich funktional für die engere Zusammenarbeit in der Eurozone notwendig droht damit eine Union in der Union zu entstehen.

Diese Verschiebung im institutionellen Gleichgewicht hin zum Ratssystem mit eigenen Doppelstrukturen für die Eurozone ist auch aus demokratisch-legitimatorischer Perspektive problematisch. Das Europäische Parlament droht dabei der Verlierer der differenzierten Integration rund um die Eurozone zu werden. In der kommenden Weiterentwicklung der Governance-Strukturen der Wirtschafts- und Währungsunion ist daher eine stärkere Beteiligung des Europäischen Parlaments notwendig, möglichst in enger Abstimmung mit den nationalen Parlamenten. Dabei wird das Parlament einen Weg finden müssen, seine Strukturen so anzupassen, dass es für Fragen der Eurozone repräsentative Entscheidungen treffen kann. Nicht zuletzt hieran wird sich entscheiden, ob es dem politischen System der Europäischen Union gelingt, weiterhin trotz zunehmender Differenzierung die Integrität der Union von bald 28 Mitgliedstaaten aufrechtzuerhalten und mit den Reformen auch die supranationale Ebene der Europäischen Union zu stärken, oder ob sich die in der Krise ausgebildete Parallelstruktur im Rat vollständig zu einer Union in der Union ausbildet. 